\title{
Zn $\begin{array}{ll}\text { Research Square } & \text { They should not be considered conclusive, used to inform clinical practice, } \\ \text { or referenced by the media as validated information. }\end{array}$
}

\section{Attitudes of Medical Workers Toward Artificial Intelligence in Ophthalmology: A Comparative Survey}

\section{Bo Zheng}

School of Infomation Engeneering of Huzhou University

\section{Maonian Wu}

School of Information Engineering of Huzhou University

Shaojun Zhu

School of Information Engineering of Huzhou Univeristy

Hongxia Zhou

School of Information Engineering of Huzhou University

\section{Xiulan Hao}

School of Information Engineering of Huzhou University

\section{Fangqin Fei}

First Affiliated Hospital of Huzhou University

\section{Yun Jia}

School of Medicine of Huzhou University

\section{Jian Wu}

Zhejiang University Real Doctor Al Research Center

\section{Xueping Pan}

First People's Hospital of Huzhou

Weihua Yang ( $\sim$ benben0606@139.com )

affiliated eye hospital of Nanjing medical universiy https://orcid.org/0000-0002-7629-0193

\section{Research article}

Keywords: artificial intelligence; ophthalmic artificial intelligence; artificial intelligence in ophthalmology; artificial intelligence in medicine; replacing doctors; doctor replacement; medical ethics

Posted Date: February 9th, 2021

DOl: https://doi.org/10.21203/rs.3.rs-195130/v1

License: (c) (1) This work is licensed under a Creative Commons Attribution 4.0 International License. Read Full License 


\section{Abstract}

Background: In the development of artificial intelligence in ophthalmology, the ophthalmic Al-related recognition issues are prominent, but there is a lack of research into people's familiarity with and their attitudes toward ophthalmic Al. This survey aims to assess medical workers' and other professional technicians' familiarity with, attitudes toward, and concerns about Al in ophthalmology.

Methods: An electronic questionnaire was designed through the app Questionnaire Star, and was sent to participants through WeChat, China's version of Facebook or WhatsApp. The participation was voluntary and anonymous. The questionnaire consisted of four parts, namely the participant's background, their basic understanding of Al, their attitudes toward Al, and their concerns about Al. A total of 562 participants were counted, with 562 valid questionnaires returned. The results of the questionnaires are displayed in an Excel 2003 form.

Results: A total of 562 participants completed the questionnaire, of whom 291 were medical workers and 271 were other professional technicians. About 1/3 of the participants understood Al and ophthalmic Al. The percentages of people who understood ophthalmic Al among medical workers and other professional technicians were about $42.61 \%$ and $15.6 \%$, respectively. About $66.01 \%$ of the participants thought that $\mathrm{Al}$ in ophthalmology would partly replace doctors, with about $59.07 \%$ still having a relatively high acceptance level of ophthalmic Al. Meanwhile, among those with Al in ophthalmology application experiences (30.6\%), above $70 \%$ of participants held a full acceptance attitude toward $\mathrm{Al}$ in ophthalmology. The participants expressed medical ethics concerns about Al in ophthalmology. And among the participants who understood Al in ophthalmology, almost all the people said that there was a need to increase the study of medical ethics issues in the ophthalmic Al field.

Conclusions: The survey results revealed that the medical workers had a higher understanding level of $\mathrm{Al}$ in ophthalmology than other professional technicians, making it necessary to popularize ophthalmic Al education among other professional technicians. Most of the participants did not have any experience in ophthalmic Al but generally had a relatively high acceptance level of Al in ophthalmology, and there was a need to strengthen research into medical ethics issues of the field.

\section{Background}

In recent years, with the increase of computational speed, neural networks have regained prosperity after hitting its rock bottom. The deep convolutional neural network AlexNet [1] winning the first place in the ImageNet competition has led to the rapid development of deep learning technology. After that, deep learning network models [2-10] have emerged generation after generation, which has accelerated the development of artificial intelligence (Al) technology. Through Al, researchers can make the preliminary diagnosis of skin cancers, achieve rapid intraoperative diagnosis of brain tumors, diagnose 55 common diseases in pediatrics based on electronic medical records in Chinese, identify rare genetic diseases through facial photographs, and generate the findings that early and frequent patient movements can 
reduce the risk of post-intensive care syndrome and long-term dysfunction after analyzing patients' movement activities in the intensive care units. [11-15]

Medical instruments are frequently used in clinical examinations in modern medicine, and imaging equipment is an important part. Imaging equipment is used for X-ray imaging, magnetic resonance imaging, ultrasound imaging, tomography imaging, and fundus photography, etc. The data in the ophthalmology field are both in a wide variety and in large quantities, among which the most common types are fundus photographs and optical coherence tomography (OCT) images, making it the most extensively researched field with Al. Fundus photography and OCT are regular examinations used in ophthalmology, through which a vast amount of high-quality standard images can be obtained. These images are suitable for analysis and process by Al deep learning technology to further assist doctors in diagnosing ophthalmopathies. Using deep learning technology in Al, Google researchers have created an algorithm that can detect diabetic retinopathy and macular edema. By analyzing human retinal images, this algorithm can very accurately predict risk factors affecting cardiovascular health. [16-17] The researchers from Sun Yat-sen University in China have developed a deep learning model called CC-Cruiser for recognizing congenital cataract, which is also able to diagnose blinding diseases such as age-related macular degeneration and diabetic macular edema after trained with deep learning algorithms based on OCT images [18-19]. Besides, there are related studies that use Al technology for the segmentation of ophthalmic images [20-21], and the classification of ophthalmopathies [22-24], etc.

Artificial intelligence technology has been studied so extensively in ophthalmology that some even have been on the commercializing stage [25], thus leading to some people believing that Al may be able to replace doctors. Some researchers investigated the attitudes of medical undergraduates to the application of Al in radiology and medicine [26], the attitudes of medical students in other regions to Al [27], the influence of artificial intelligence on radiology [28], as well as how to cope with the ethical challenges in medical Al [29-30], etc. However, few people know about medical workers' familiarity with and their attitudes toward Al in ophthalmology. For this purpose, a questionnaire was designed to assess medical workers' (health care workers or medical students) understanding level of and their attitudes toward Al. Meanwhile, the questionnaire also surveyed other professional technicians using the same questions as a comparison.

The survey of $\mathrm{Al}$ in ophthalmology-related questions cannot only evaluate the attitudes of medical workers (health care workers or medical students) and other professional technicians toward Al and clarify the dilemma facing the current technological development, it can also provide theoretical guidance for its future practice and application. At present, in the development of $\mathrm{Al}$ in ophthalmology, there are a lot of existing and foreseeable medical ethical problems. Analysis of the causes of these problems through this survey may help to put forward corresponding countermeasures so that we can draw on advantages and avoid disadvantages in the future development of Al in ophthalmology.

\section{Methods}


Using the Questionnaire Star APP, we designed an electronic questionnaire that consisted of four parts. The first part was the participant's basic information, including the participant's gender, age, educational level, place of residence, work area and professional title; the second part was about the participant's basic understanding of $\mathrm{Al}$, including whether the participant understood $\mathrm{Al}$, medical $\mathrm{Al}$, and $\mathrm{Al}$ in ophthalmology, as well as the participant's evaluation of the current development of Al in ophthalmology; the third part was about the participant's attitude to Al, including whether he/she thought $\mathrm{Al}$ in ophthalmology would replace doctors, whether he/she had experience in Al in ophthalmology application, and his/her acceptance level of Al in ophthalmology; and the fourth part was about the participant's concerns about Al, which included the participant' specific concerns about Al in ophthalmology and whether he/she thought it was necessary to strengthen medical ethics research in the field.

Participants of the questionnaire were mainly members of the Zhejiang Society of Mathematical Medicine, with their locations covering various cities and counties mainly in Zhejiang Province. They worked as ophthalmologists, medical students, Al technicians, and professional technicians in other fields. Their educational levels were above junior high school and could understand the questionnaire well. The questions in the questionnaire have been investigated, sorted and summarized repeatedly in a broad and deep way. The survey is a targeted group survey. Questionnaires were sent to medical workers through professional ophthalmological or medical intelligence groups and other professionals through related professional groups. Before we collect the questionnaires for statistical analysis, each participant was invited to fill out the questionnaire once based on a voluntary and anonymous principle and was informed that the results of the survey would be further used for statistical assessment and publication. A total of 562 participants were counted, with 562 valid questionnaires returned. The results of the questionnaires are displayed in an Excel 2003 form.

According to the Article 3 of the Measures for the Ethical Review of Biomedical Research Involving Humans issued by the National Health and Family Planning Commission in 2016, ethical review is unnecessary for the study.

\section{Results}

\section{Basic information of participants}

Of the 562 participants, 291 were medical workers $(51.78 \%)$ with the rest being other professional technicians (48.22\%). Nearly half of them were from prefecture-level cities (47.69\%), about 1/4 (24.91\%) were from provincial capital cities, and the remaining were from other regions $(27.41 \%) .9 .96 \%$ had a doctor's degree or higher education, $12.99 \%$ had a master's degree, and $77.05 \%$ had a bachelor's degree or lower education; $31.49 \%$ had senior titles, $25.44 \%$ were with intermediate titles, and $32.03 \%$ were primary and ungraded professionals. The complex structure of these participants had relatively good social representative value.

\section{Participants' basic understanding of artificial intelligence}


The percentage of participants who completely understood and almost understood Al was $37.9 \%$, who understood a little was $52.14 \%$, and who didn't understand Al was $9.96 \%$; the percentage of participants who completely understood and almost understood medical Al was $31.67 \%$, who understood a little was $44.31 \%$ and who didn't understand medical Al was $24.02 \%$; the percentage of participants who completely understood and almost understood Al in ophthalmology was $29.54 \%$, who understood a little was $34.52 \%$, and who didn't understand $\mathrm{Al}$ in ophthalmology was $35.94 \%$. That is to say, the number of participants whose understanding level of $\mathrm{Al}$, medical Al, and $\mathrm{Al}$ in ophthalmology was "completely", "almost" or "a little" was gradually decreasing in the mentioned order (Al, medical Al, and ophthalmological Al), while the number of people who did not know about Al, medical Al, and Al in ophthalmology was gradually increasing in the same order.

Among the participants, $19.4 \%$ thought that the current development of Al in ophthalmology was very good. $42.7 \%$ thought that its current development was good. $35.23 \%$ thought that its current development was average. While the remaining $2.67 \%$ thought that the development level was poor. Therefore, the vast majority of the participants held a positive or wait-and-see attitude toward the current development of $\mathrm{Al}$ in ophthalmology, with a few others having a negative attitude.

Table 3, Fig. 1, Fig. 2, and Fig. 3 show that the numbers of medical workers whose understanding level was "almost understand" or above (including "completely understand" and "almost understand") were $47.42 \%, 45.02 \%$, and $42.61 \%$, respectively, for Al, medical Al, and $\mathrm{Al}$ in ophthalmology. For other professional technicians, the numbers were $27.68 \%, 17.35 \%$, and $15.5 \%$, respectively. For the two different groups by working fields, the participants' understanding level of Al, medical Al, and Al in ophthalmology tended to drop in the mentioned order. But the number of people whose understanding level of these three Als was "almost understand" and above was greater among medical workers than among other professional technicians. Artificial intelligence is relatively well popularized in the medical field, meanwhile, there is a need to enhance the popularization of artificial intelligence-related knowledge among people in other fields. 
Table 1

Participants' basic information ( $N=562)$.

\begin{tabular}{|c|c|}
\hline Characteristic & Participants \\
\hline \multicolumn{2}{|l|}{ Gender, $n(\%)$} \\
\hline Male & $216(38.43)$ \\
\hline Female & $346(61.57)$ \\
\hline \multicolumn{2}{|l|}{ Age (years), n(\%) } \\
\hline 25 or less & $70(12.46)$ \\
\hline $25-45$ & $340(60.5)$ \\
\hline 45 or more & 152(27.05) \\
\hline \multicolumn{2}{|l|}{ Work area, n(\%) } \\
\hline Medical worker (health care worker or medical student) & 291(51.78) \\
\hline Other professional technicians & $271(48.22)$ \\
\hline \multicolumn{2}{|l|}{ Place of residence, $\mathrm{n}(\%)$} \\
\hline Provincial capital & $140(24.91)$ \\
\hline Prefecture-level city & 268(47.69) \\
\hline County and below & $152(27.05)$ \\
\hline Abroad & 2(0.36) \\
\hline \multicolumn{2}{|l|}{ Education, n(\%) } \\
\hline Doctor and above & $56(9.96)$ \\
\hline Master & $73(12.99)$ \\
\hline Bachelor & $244(43.42)$ \\
\hline Other lower education & 189(33.63) \\
\hline \multicolumn{2}{|l|}{ Professional title, $n(\%)$} \\
\hline Ungraded & $180(32.03)$ \\
\hline Primary & $62(11.03)$ \\
\hline Intermediate & $143(25.44)$ \\
\hline Senior & 177(31.49) \\
\hline
\end{tabular}


Table 2

Participants' basic understanding of artificial intelligence $(N=562)$.

\begin{tabular}{|ll|}
\hline Understanding level & Value \\
\hline Artificial intelligence, $n(\%)$ & $39(6.94)$ \\
\hline Completely & $174(30.96)$ \\
\hline Almost & $293(52.14)$ \\
\hline A little & $56(9.96)$ \\
\hline Not understand & \\
\hline Medical artificial intelligence, $n(\%)$ & $33(5.87)$ \\
\hline Completely & $145(25.8)$ \\
\hline Almost & $249(44.31)$ \\
\hline A little & $135(24.02)$ \\
\hline Not understand & \\
\hline Ophthalmic artificial intelligence, $n(\%)$ & $34(6.05)$ \\
\hline Completely & $132(23.49)$ \\
\hline Almost & $194(34.52)$ \\
\hline A little & $202(35.94)$ \\
\hline Not understand & $109(19.4)$ \\
\hline Current development of ophthalmic artificial intelligence, $n(\%)$ & \\
\hline Very good & $15(2.67)$ \\
\hline Good & \\
\hline Average & \\
\hline Poor & \\
\hline
\end{tabular}


Table 3

Medical workers and other professional technicians ' basic understanding of artificial intelligence $(\mathrm{N} 1=291, \mathrm{~N} 2=271)$.

\begin{tabular}{|lll|}
\hline Understanding level & Value1 & Value2 \\
\hline Artificial intelligence, $\mathbf{n}(\%)$ & & \\
\hline Completely & $17(5.84)$ & $22(8.12)$ \\
\hline Almost & $121(41.58)$ & $53(19.56)$ \\
\hline A little & $140(48.11)$ & $153(56.46)$ \\
\hline Not understand & $13(4.47)$ & $43(15.86)$ \\
\hline Medical artificial intelligence, $\mathbf{n ( \% )}$ & & \\
\hline Completely & $18(6.19)$ & $15(5.54)$ \\
\hline Almost & $113(38.83)$ & $32(11.81)$ \\
\hline A little & $132(45.36)$ & $117(43.17)$ \\
\hline Not understand & $28(9.62)$ & $107(39.48)$ \\
\hline Ophthalmic artificial intelligence, $\mathrm{n}(\%)$ & \\
\hline Completely & $19(6.53)$ & $15(5.54)$ \\
\hline Almost & $105(36.08)$ & $27(9.96)$ \\
\hline A little & $121(41.58)$ & $73(26.94)$ \\
\hline Not understand & $46(15.81)$ & $156(57.56)$ \\
\hline
\end{tabular}

As is shown in Fig. 4, about $71 \%$ of medical workers and $53 \%$ of other professional technicians believed that the current development of $\mathrm{Al}$ in ophthalmology was good; while among those who completely understood and almost understood $\mathrm{Al}$ in ophthalmology, about $81 \%$ of medical workers and $76 \%$ of other professional technicians thought that the current development of Al in ophthalmology was good.

\section{Participants' attitudes toward artificial intelligence}


In Table 4, on the question of whether Al in ophthalmology would replace doctors, $24.02 \%$ of participants said no. But $66.01 \%$ thought it would partly replace doctors, and $1.78 \%$ thought that Al in ophthalmology had a bright prospect and would completely replace doctors. As is shown in Fig. 6, about $97 \%$ of medical workers and $85 \%$ of other professional technicians believed that Al in ophthalmology would partly or would not replace doctors, suggesting that the participants, while acknowledging $\mathrm{Al}$ in ophthalmology, also recognized the value of ophthalmologists.

Table 4

Participants' attitudes toward artificial intelligence $(\mathrm{N}=562)$.

\begin{tabular}{|ll|}
\hline Al in ophthalmology will replace ophthalmologists, $\mathbf{n}(\%)$ & \\
\hline Completely & $10(1.78)$ \\
\hline Partly & $371(66.01)$ \\
\hline Not & $135(24.02)$ \\
\hline Don't understand & $46(8.19)$ \\
\hline Do you have any experience with Al in ophthalmology?, $\mathbf{n}(\%)$ & \\
\hline Being using or about to use & $29(5.16)$ \\
\hline Having applied & $49(8.72)$ \\
\hline Having experienced & $94(16.73)$ \\
\hline Having no related experience & $390(69.4)$ \\
\hline Acceptance level of Al in ophthalmology, $\mathbf{n}(\%)$ & \\
\hline Completely & $332(59.07)$ \\
\hline Partly & $218(38.79)$ \\
\hline Not accept & $10(1.78)$ \\
\hline Strongly resist & $2(0.36)$ \\
\hline
\end{tabular}

$69.4 \%$ of the participants had no ophthalmic Al-related experience while the rest had applied or experienced Al in ophthalmology. As is shown in Fig. 7, 56.36\% of medical workers and $83.39 \%$ of other professional technicians had no experience in the application of $\mathrm{Al}$ in ophthalmology, indicating that while developing rapidly, Al in ophthalmology hasn't been widely used.

$59.07 \%$ of the participants had a relatively high acceptance level of Al in ophthalmology, with only $2.14 \%$ against it. As is shown in Fig. 8, among those with experience in the application of Al in ophthalmology, $84.25 \%$ of medical workers and $73.33 \%$ of other professional technicians would fully accept it.

\section{Participants' concerns about artificial intelligence}


In Table 5, among the participants, 56.41\% said that in the current ophthalmic Al practice, medical responsibilities are unclear; $49.29 \%$ said that the quality of $\mathrm{Al}$ in ophthalmology services was difficult to guarantee; while the percentage of those who thought there existed problems such as extreme high prices, medical ethical risks and lack of political support was about $40 \%$. More than $90 \%$ of the participants thought there was a need to strengthen medical ethics research in the ophthalmic Al field. Among those who completely and almost understood $\mathrm{Al}$ in ophthalmology, $98.39 \%$ of medical workers and $95.24 \%$ of other professional technicians believed it was necessary to strengthen medical ethics research in the field, as is shown in Fig. 9. This is enough to showcase the importance of addressing medical ethical issues in the ophthalmic Al field.

Table 5

Participants' concerns about artificial intelligence $(\mathrm{N}=562)$.

\section{What are your concerns about Al in ophthalmology? (multiple selection), $\mathrm{n}(\%)$}

Medical responsibilities are unclear

$317(56.41)$

Service price is too expensive

252(44.84)

Service quality is difficult to guarantee

277(49.29)

Medical ethical risk

240(42.7)

Policy support may not be in place

235(41.81)

Others

85(15.12)

Is it necessary to strengthen the study of medical ethics in the ophthalmic Al field?, $n(\%)$

Yes

517(91.99)

No

$45(8.01)$

\section{Discussion}

In the survey, the number of medical workers participated was roughly equal to that of other professional technicians. The proportion of participants who did not understand Al among other professional technicians was about three times that of medical workers. And the proportion of those who didn't understand medical Al among other professional technicians was about 4 times that of medical workers. The proportion of people who did not understand Al in ophthalmology among other professional technicians was about four times that of medical workers. Hence one can see that the understanding level of Al, medical Al, and ophthalmic Al among the medical workers who participated in the survey is higher than that among other professional technicians, suggesting that in China, $\mathrm{Al}$ is relatively more widely popularized among medical workers. In recent years, Al has been more and more widely introduced in the medical field. Due to work or academic exchanges, medical workers have more 
opportunities to learn about medical Al than other professional technicians. The other professional technicians in this survey were technical personnel whose research areas had no connection to medicine or Al. As a result, they had relatively less knowledge about medical Al and fewer opportunities to apply or experience medical Al, especially in the more specialized clinical ophthalmology field. Naturally, those in the survey who had no medical background would have a difficult time imagining the application of $\mathrm{Al}$ in medicine. Therefore, the real-life application of Al in other areas should be further popularized in our society to gain more of people's trust before Al can be used in medical care.

Concerning the attitudes toward Al, both medical workers and other professional technicians were relatively confident in human doctors, with only a very small number of people thinking that $\mathrm{Al}$ in ophthalmology would completely replace ophthalmologists. Most participants were relatively rational, believing that ophthalmic Al would only partly replace ophthalmologists. As people become more rational about $\mathrm{Al}$ in ophthalmology, the condition has been more and more favorable for the healthy development of $\mathrm{Al}$ in the medical field. As suggested by Turing for the healthy development of Al, "Instead of trying to produce a program to simulate the adult mind, why not rather try to produce one which simulates the child's? If this were then subjected to an appropriate course of education one would obtain the adult brain." [31] This conception has functioned as guidance in the research of medical Al. Whether it's Nature's publication of Al used for DR diagnosis or the U.S. Food and Drug Administration's approval of IDx-DR in 2018 [25], there has been no real diagnostic systems that can fully diagnose and identify all the 4 grades of DR, which means there is still a long way for ophthalmic Al to go from laboratory research to clinical application. But undeniably, in a country such as China where ophthalmologists are in much dire need (the over 1.4 billion Chinese people only share about 36,000 ophthalmologists), the application of $\mathrm{Al}$ can help to diagnose and treat many more patients, which would reduce the burden on ophthalmologists, thus probably having led to the higher acceptance level of ophthalmic Al among medical workers. Meanwhile, those other professional technicians, despite a relatively lower understanding level, also basically held a positive attitude to acknowledging Al in ophthalmology.

As far as the application of ophthalmic Al goes, there are currently some pilot centers in China that provide opportunities to experience its real-life application. However, due to the rarity of such centers, only close to half of the medical workers involved in the survey had related experience while $4 / 5$ of the other professional technicians had no such experience. This indicates that the application of $\mathrm{Al}$ in ophthalmology is not yet popularized. It has very few real-life applications, mainly due to technical and ethical issues. On the technical side, the systems that had relatively good diagnosing abilities turned out to be not well performed in the complex real-life scenarios, requiring further improvement. And on the ethics side, the medical responsibilities are not clearly defined for the artificial intelligence diagnostic systems and there are no related policies to follow or to regulate it with. Therefore, although the research of $\mathrm{Al}$ in ophthalmology is going well, it still takes prudence in its real-life application.

In recent years, the application of Al in ophthalmology is very deeply researched, but there are not so many studies on related policies and ethics. The survey found that unclear medical responsibilities and difficulty in guaranteeing service quality respectively ranked as the No. 1 and No. 2 concerns about the 
use of $\mathrm{Al}$ in ophthalmology, with $60 \%$ of the participants worrying about the "unclear medical responsibilities." These concerns are sufficient to show that the country needs to improve its regulation system of Al and strengthen the exploration of relevant medical ethics issues. Only when the relevant regulation system and ethics issues have been addressed can we guarantee the real-life practice of medical Al, and establish people's confidence in medical Al so that they can truly accept its relevant application.

The survey mainly analyzed the participants' understanding and acceptance level of Al in ophthalmology, as well as the participants' concerns about Al in ophthalmology. The participants in this survey are mainly from Zhejiang, China, an important part of the Yangtze River Delta, which is one of china's relatively developed regions. With almost no participants from remote areas, the survey results do not represent those of medical workers and other professional technicians nationwide. Besides, although the participants are relatively evenly distributed among the different groups, the total number is relatively small. That is to say, the follow-up surveys should try to broaden the survey scope, making the findings more credible and broadly representative.

\section{Conclusions}

The survey results revealed that the medical workers had a higher understanding level of $A l$ in ophthalmology than other professional technicians, making it necessary to popularize ophthalmic Al education among other professional technicians. Most of the participants did not have any experience in ophthalmic Al, but generally had a relatively high acceptance level of Al in ophthalmology, and there was a need to strengthen research into the medical ethics issues of the field.

\section{Abbreviations}

$\mathrm{Al}$

artificial intelligence

OCT

optical coherence tomography

DR

diabetic retinopathy

\section{Declarations}

\section{Ethics approval and consent to participate}

In China, the National Health and Family Planning Commission officially issued Measures for the Ethical Review of Biomedical Research Involving Humans in 2016. The Article 3 defines the biomedical research involving human subjects.

Article 3. Biomedical research involving human subjects in the Measures includes: 
(1) activities of conducting research on human physiology, psychological behavior, pathological phenomena, causes and pathogenesis of diseases, as well as the prevention, diagnosis, treatment and rehabilitation of diseases, using modern methods in physics, chemistry, biology, traditional Chinese medicine and psychology, etc.;

(2) activities of experimental research on the human body of new medical technologies or new medical products;

(3) activities in which scientific research materials such as specimens, medical records and behaviors relating to humans are collected, recorded, used, reported or stored by epidemiological, sociological and psychological methods.

This study mainly investigates people's current understanding of the cross field application of artificial intelligence in medicine, which does not involve the activities stipulated in Article 3 of the censorship measures. Thus ethical review is not required for the study.

Participants were informed verbally as well as in written form in the questionnaire that completing and submitting the questionnaire meant they agreed to participate in the study.

\section{Consent for publications}

Participants were verbally informed that the results of the survey would be further used for statistical assessment and publication, which is also clearly stated in written form in the questionnaire.

\section{Availability of data and materials}

All data generated or analyzed during this study are included in this published article.

\section{Competing interests}

The authors declared no competing interests.

\section{Funding}

The Natural Science Foundation of Zhejiang Province (LQ18F020002) mainly studies DR images labeling and diagnosis based on it. The Zhejiang Basic Public Welfare Research Program (LGF18H120003) mainly studies intelligent diagnostic systems of DR. The National Natural Science Foundation of China (61906066) mainly studies vision prediction after cataract surgery. The Zhejiang Medical and Health Research Project (2018270516) and the Huzhou Science and Technology Planning Program (2019YSR23) mainly study the cross field application and promotion of artificial intelligence and ophthalmological diagnostic technologies. All the above foundations focus on the cross field application 
of artificial intelligence in medicine (especially in ophthalmology) and need to know about people's knowledge of artificial intelligence, artificial intelligence in medicine and artificial intelligence in ophthalmology, which is why the support for the study.

\section{Authors' contributions}

During the study, all authors have contributed significantly. WY and BZ, FF analyzed data and drafted the manuscript. MW and SZ, $\mathrm{HZ}$ analyzed data and revised the draft. $\mathrm{WY}, \mathrm{BZ}, \mathrm{MW}, \mathrm{XH}, \mathrm{YJ}$ and JW designed electronic questionnaire. WY, BZ, MW, XH, and XP sent the questionnaire to participants. WY and XP designed the experiment and built initial constructs. WY, BZ and XP together proposed the idea and supervised the project.

All authors have read and approved the manuscript.

\section{Acknowledgements}

Not applicable.

\section{References}

1. Krizhevsky A, Sutskever I, Hinton GE. Imagenet classification with deep convolutional neural networks[C]//Advances in neural information processing systems. 2012: 1097-1105.

2. Simonyan $\mathrm{K}$, Zisserman A. Very deep convolutional networks for large-scale image recognition[C]//Advances in International Conference on Learning Representations. May 2015.

3. Szegedy C, Liu W, Jia Y, et al. Going deeper with convolutions[C]//Proceedings of the IEEE conference on computer vision and pattern recognition. 2015: 1-9.

4. He K, Zhang X, Ren S, et al. Deep residual learning for image recognition[C]//Proceedings of the IEEE conference on computer vision and pattern recognition. 2016: 770-778.

5. Huang G, Liu Z, Van Der Maaten L, et al. Densely connected convolutional networks[C]//Proceedings of the IEEE conference on computer vision and pattern recognition. 2017: 4700-4708.

6. Girshick R, Donahue J, Darrell T, et al. Rich feature hierarchies for accurate object detection and semantic segmentation[C]//Proceedings of the IEEE conference on computer vision and pattern recognition. 2014: 580-587.

7. Redmon J, Divvala S, Girshick R, et al. You only look once: Unified, real-time object detection[C]//Proceedings of the IEEE conference on computer vision and pattern recognition. 2016: 779-788.

8. Long J, Shelhamer E, Darrell T. Fully convolutional networks for semantic segmentation[C]//Proceedings of the IEEE conference on computer vision and pattern recognition. 2015: 3431-3440. 
9. Ronneberger O, Fischer P, Brox T. U-net: Convolutional networks for biomedical image segmentation[C]//International Conference on Medical image computing and computer-assisted intervention. Springer, Cham, 2015: 234-241.

10. Xingjian SHI, Chen Z, Wang H, et al. Convolutional LSTM network: a machine learning approach for precipitation nowcasting[C]//Advances in Neural Information Processing Systems. 2015: 802-810.

11. Esteva A, Kuprel B, Novoa RA, et al. Dermatologist-level Classification of Skin Cancer with Deep Neural Networks[J]. Nature; 2017: 115-118.

12. Orringer DA, Pandian B, Niknafs YS, et al. Rapid intraoperative histology of unprocessed surgical specimens via fibre-laser-based stimulated raman scattering microscopy[J]. Nature Boimedical Engineering; 2017: 1-13.

13. Liang $\mathrm{H}$, Tsui BY, $\mathrm{Ni} \mathrm{H}$, et al. Evaluation and accurate diagnoses of pediatric diseases using artificial intelligence[J]. Nat Med. 2019;25(2):433-8.

14. Gurovich Y, Hanani Y, Bar O, et al. Identifying facial phenotypes of genetic disorders using deep learning[J]. Nat Med. 2019;25(1):60-4.

15. Yeung S, Rinaldo F, Jopling J, et al. A computer vision system for deep learning-based detection of patient mobilization activities in the ICU[J]. npj Digital Medicine; 2019(3): 1-5.

16. Gulshan V, Peng L, Coram M, et al. Development and validation of a aeep learning algorithm for detection of diabetic retinopathy in retinal fundus photographs[J]. JAMA. 2016;316(22):2402-10.

17. Poplin R, Varadarajan AV, Blumer $K$, et al. Prediction of cardiovascular risk factors from retinal fundus photographs via deep learning[J]. Nature Biomedical Engineering. 2018;2(3):158-64.

18. Long E, Lin H, Liu Z, et al. An artificial intelligence platform for the multihospital collaborative management of congenital cataracts[J]. Nature Biomedical Engineering. 2017;1(2):1-8.

19. Kermany S, Goldbaum M, Cai W, et al. Identifying medical diagnoses and treatable diseases by image-based deep learning[J]. Cell. 2018;172(5):1122-31.

20. Pekala M, Joshi N, Liu TYA, et al. Deep learning based retinal OCT segmentation[J]. Comput Biol Med. 2019;114:103445.

21. Gao K, Niu S, Ji Z, et al. Double-branched and area-constraint fully convolutional networks for automated serous retinal detachment segmentation in SD-OCT images[J]. Computer Methods and Programs in Biomedicine; 2019, 176: 69-80.

22. Zhang H, Niu K, Xiong $\mathrm{Y}$, et al. Automatic cataract grading methods based on deep learning[J]. Computer Methods and Programs in Biomedicine; 2019, 182: 104978.

23. de La Torre J, Valls A, Puig D. A deep learning interpretable classifier for diabetic retinopathy disease grading[J]. Neurocomputing; 2019.

24. Zhao H, Sun Y, Li H. Retinal vascular junction detection and classification via deep neural networks[J]. Computer Methods and Programs in Biomedicine; 2020, 183: 105096.

25. Abràmoff MD, Lavin PT, Michele $B$, et al. Pivotal trial of an autonomous Al-based diagnostic system for detection of diabetic retinopathy in primary care offices[J]. npj Digital Medicine; 2018, 1(1): 1-8. 
26. Dos Santos DP, Giese D, Brodehl S, et al. Medical students' attitude towards artificial intelligence: a multicentre survey[J]. European Radiology; 2019, 29(4): 1640-1646.

27. Abid S, Awan B, Ismail T, et al. Artificial intelligence: medical students' attitude in district Peshawar Pakistan[J]. Pakistan Journal of Public Health. 2019;9(1):19-21.

28. Gong B, Nugent JP, Guest W, et al. Influence of artificial intelligence on Canadian medical students' preference for radiology specialty: a national survey study[J]. Academic Radiology; 2019, 26(4): 566-577.

29. Vayena E, Blasimme A, Cohen IG. Machine learning in medicine: Addressing ethical challenges[J]. PLoS Med. 2018;15(11):e1002689.

30. Keskinbora KH. Medical ethics considerations on artificial intelligence[J]. Journal of Clinical Neuroscience; 2019.

31. Turing AM. Computing machinery and intelligence[M]//Parsing the Turing Test. Dordrecht: Springer; 2009. pp. 23-65.

\section{Figures}

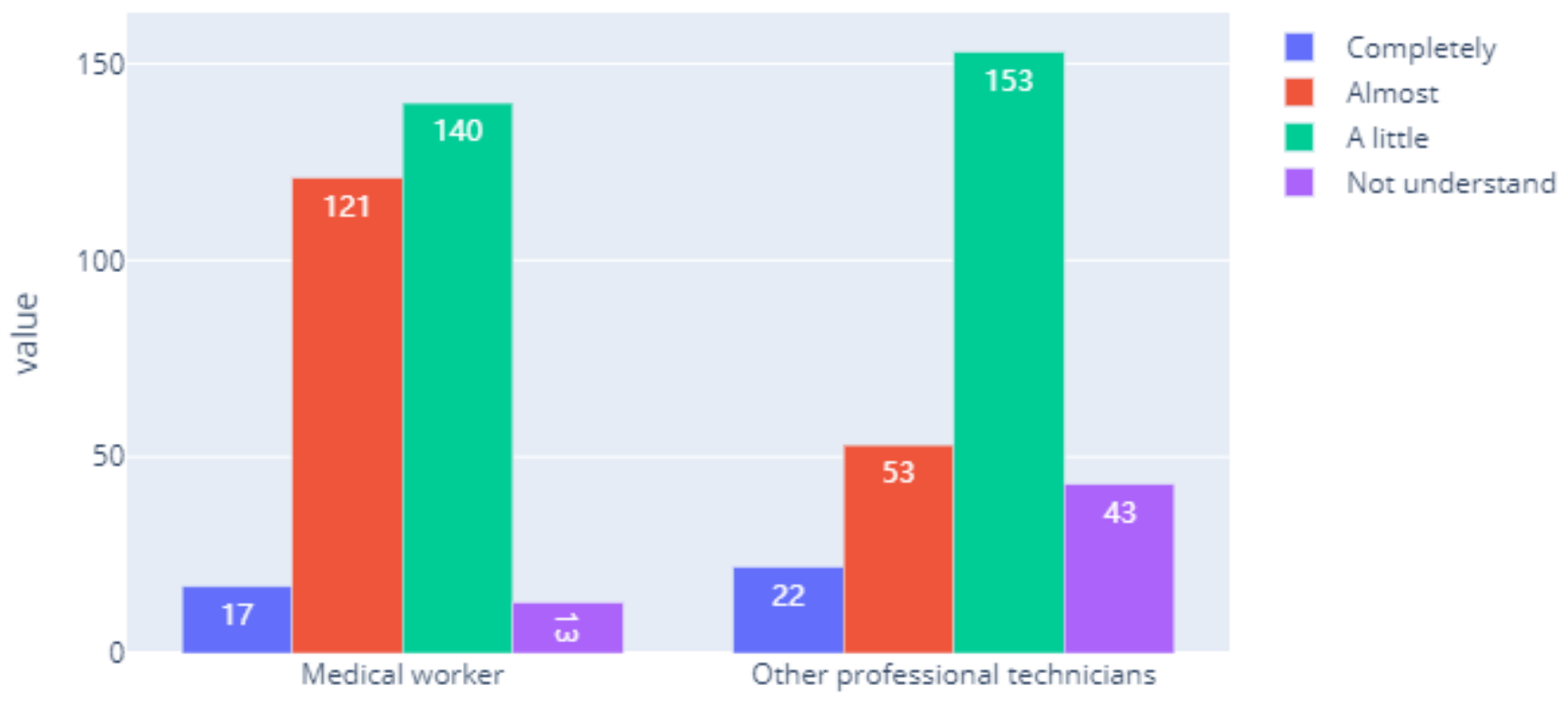

Work area

\section{Figure 1}

Participants' understanding level of artificial intelligence. 


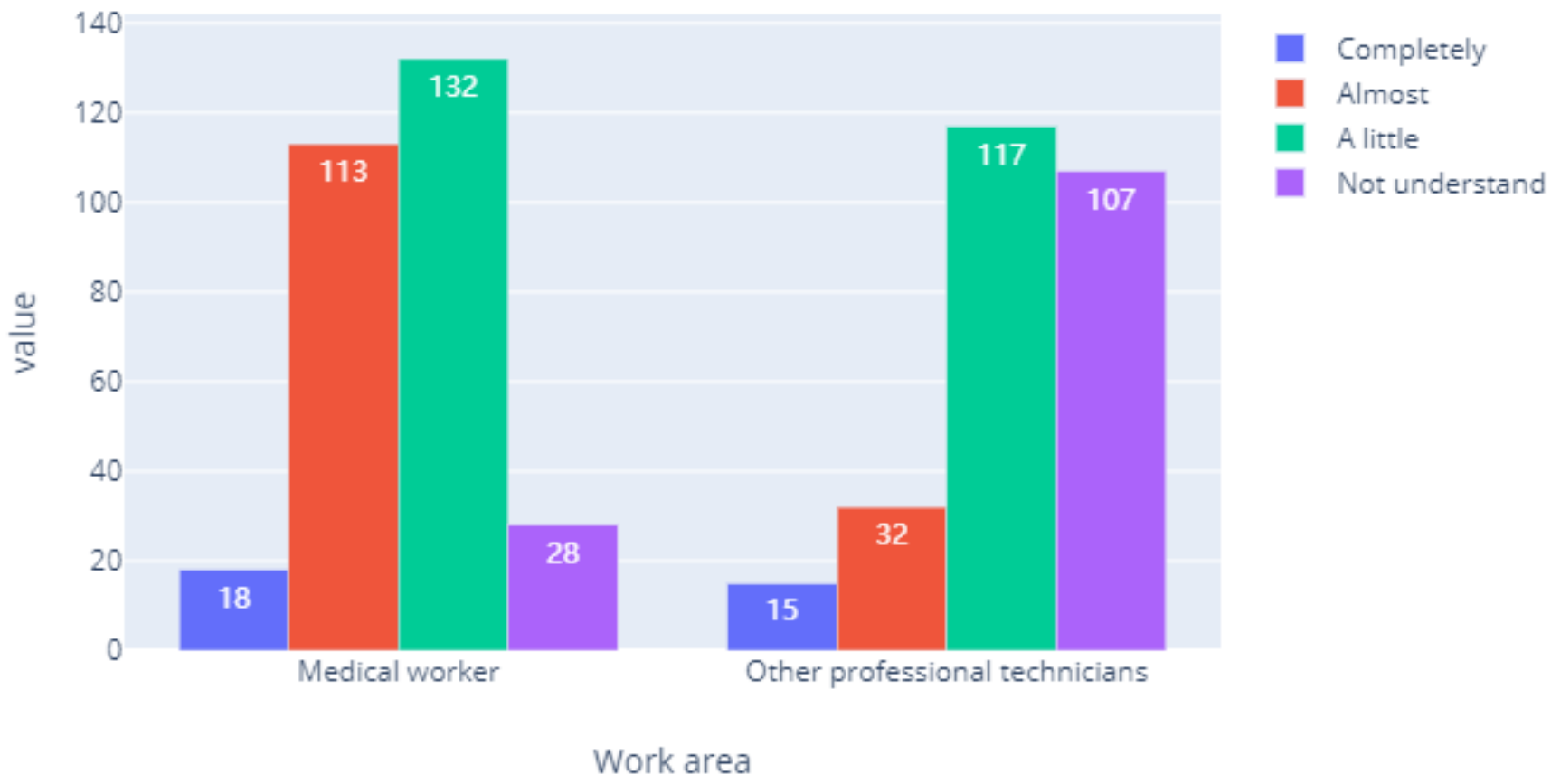

Figure 2

Participants' understanding level of medical artificial intelligence. 


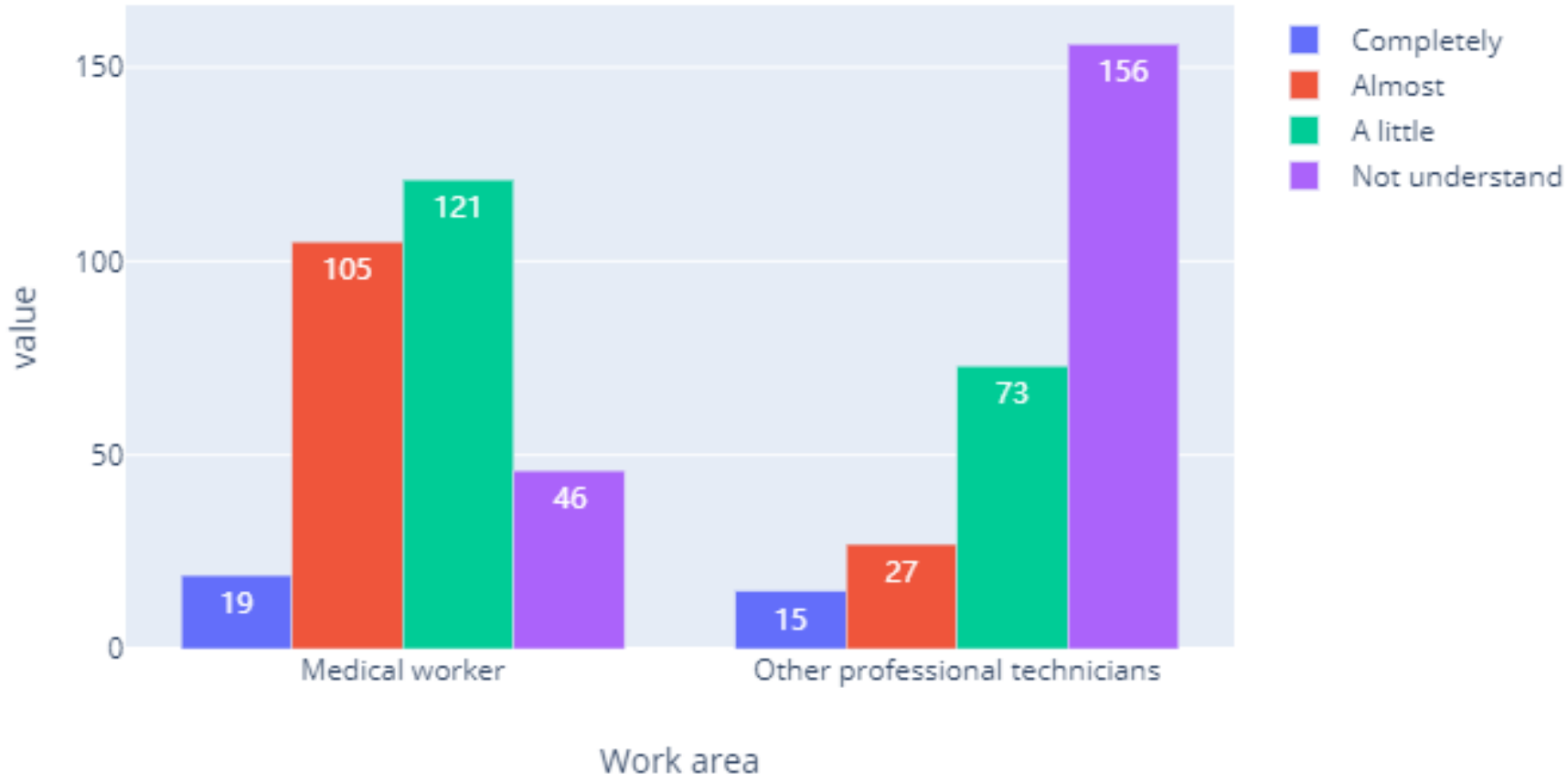

Figure 3

Participants' understanding level of ophthalmic artificial intelligence. 


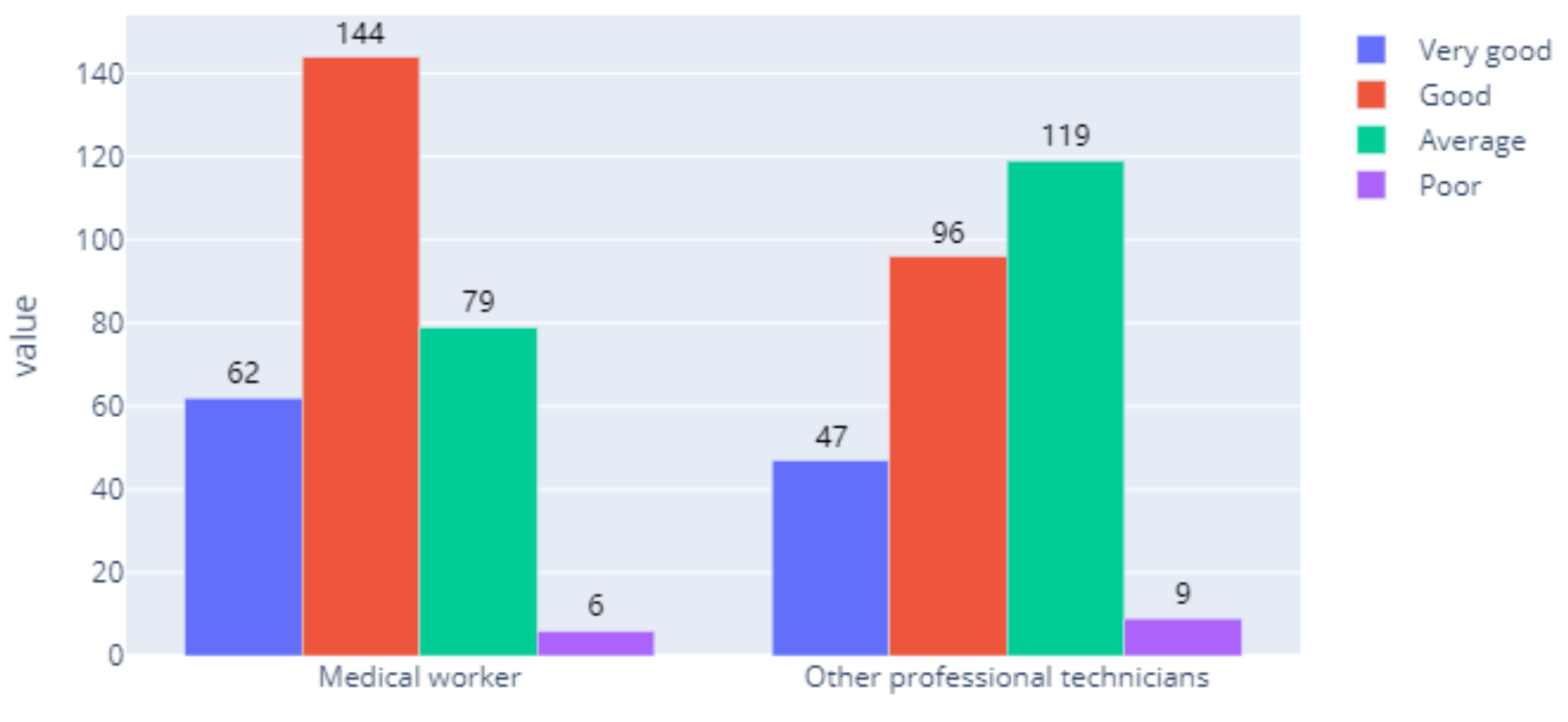

Work area

\section{Figure 4}

Participants' evaluation of the current development level of artificial intelligence. 


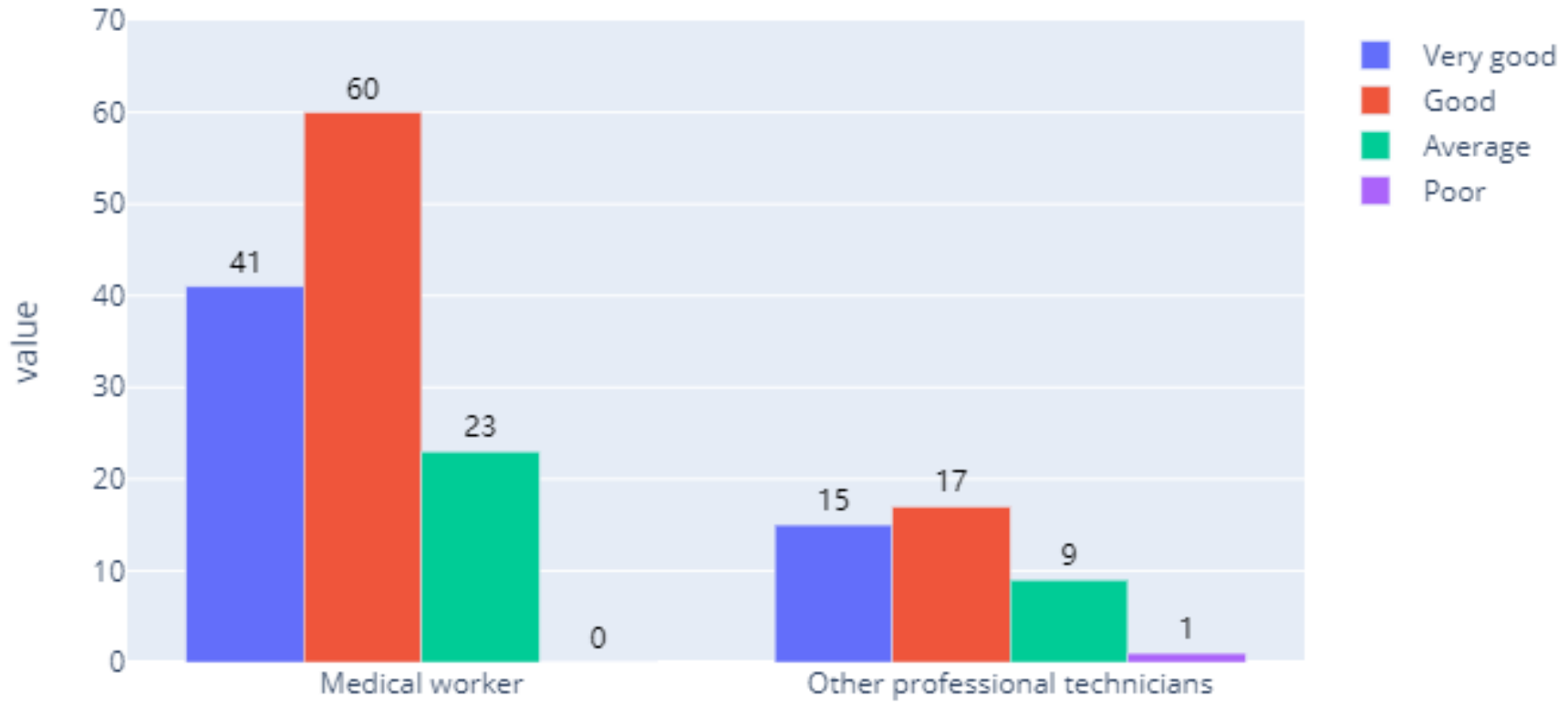

Work area

\section{Figure 5}

Evaluation of the current development level of artificial intelligence of the participants who completely and almost understood artificial intelligence.

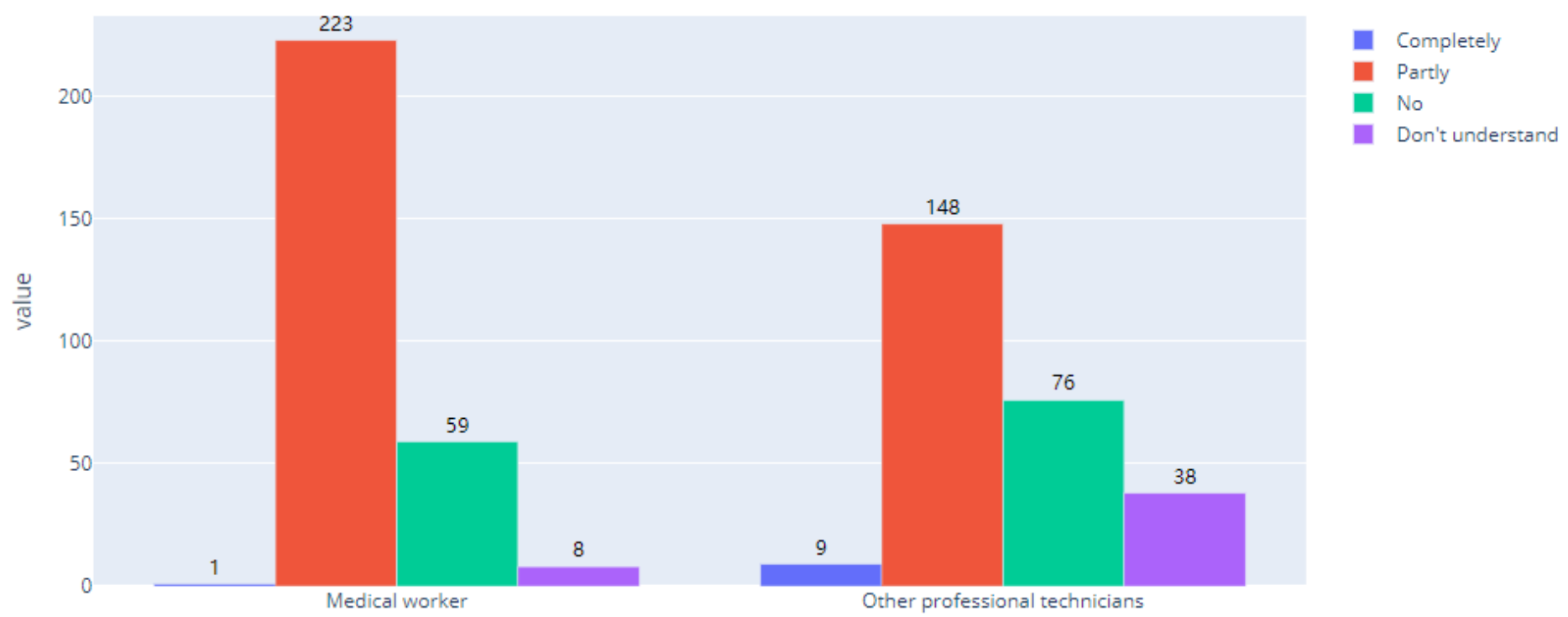

Work area 
Figure 6

Participants' prediction on the extent to which ophthalmic artificial intelligence would replace doctors.

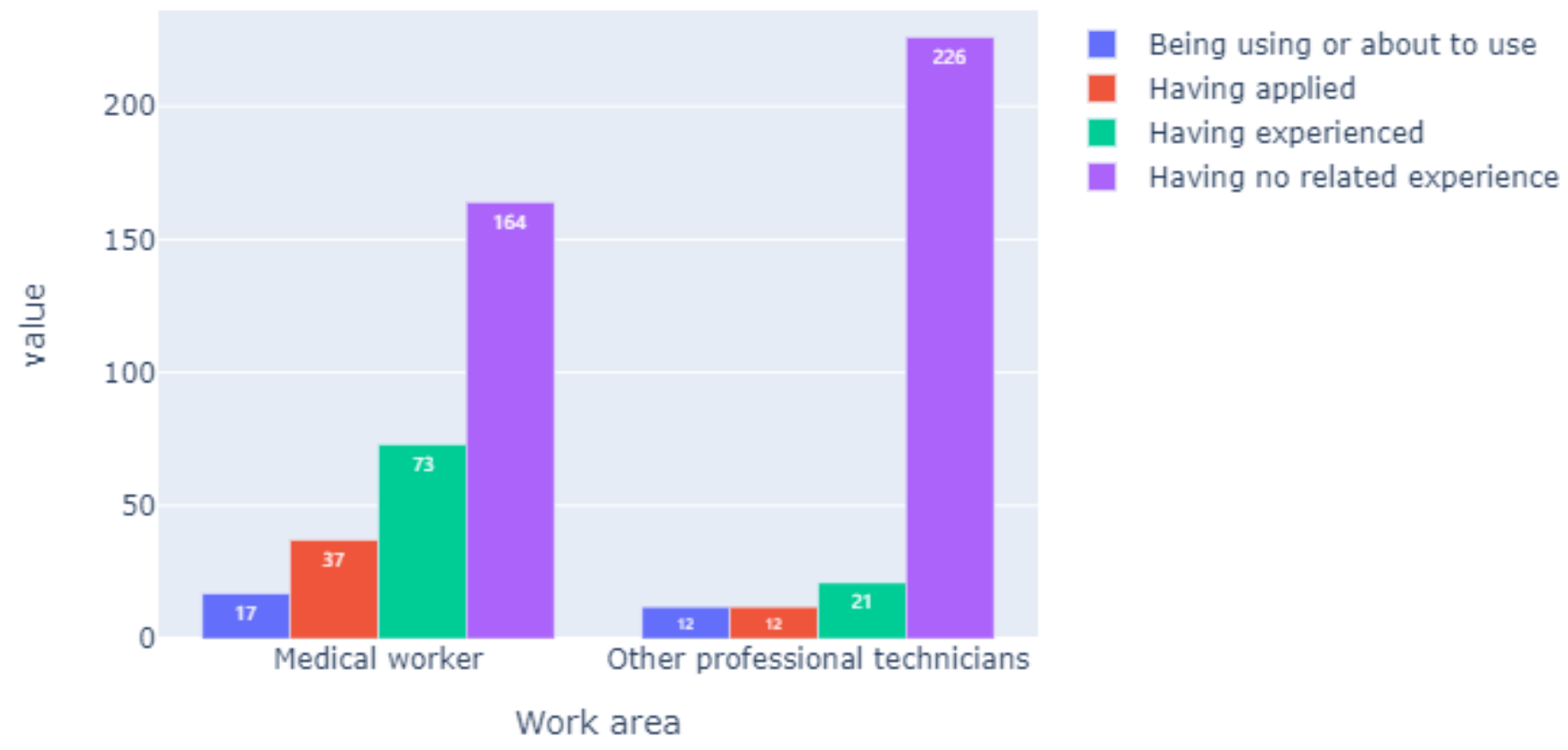

Figure 7

Participants' experience in the application of ophthalmic artificial intelligence. 


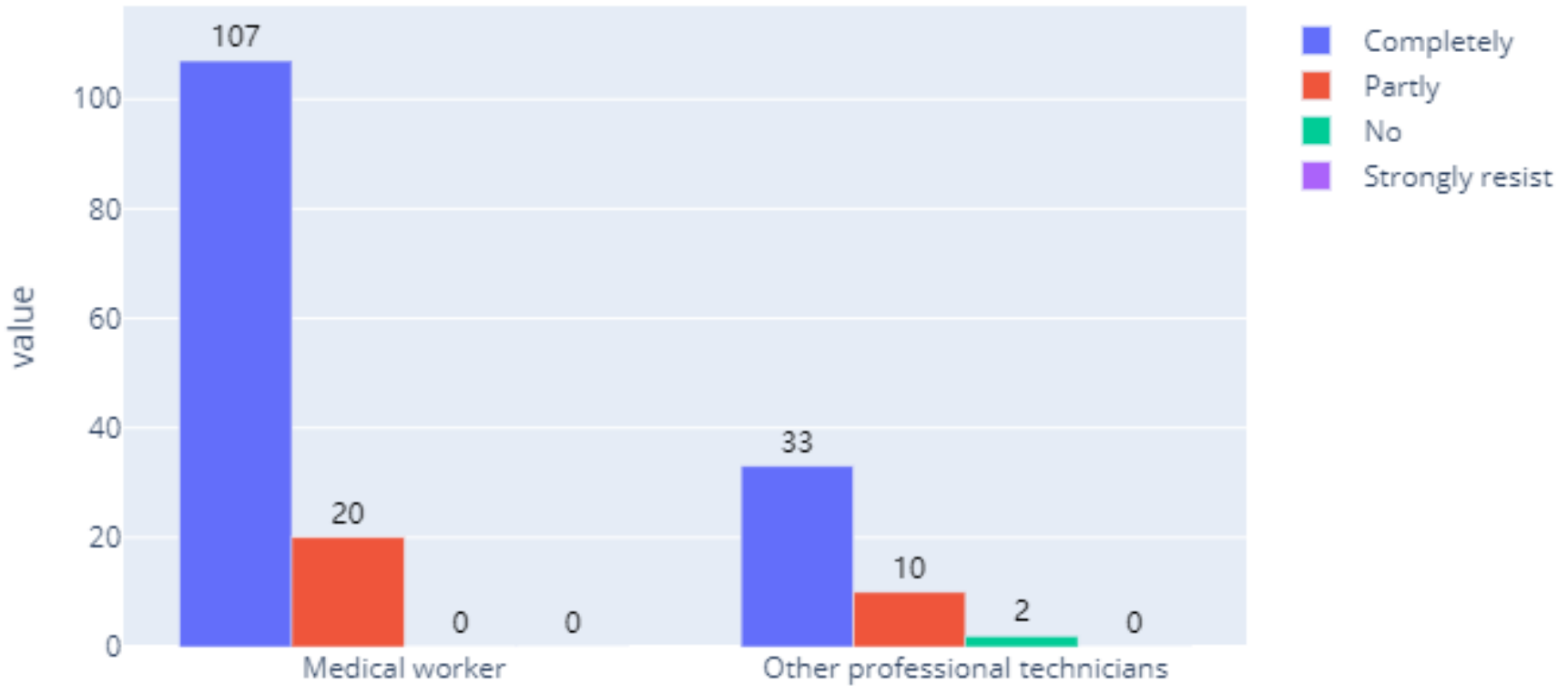

Work area

\section{Figure 8}

Acceptance level of ophthalmic artificial intelligence among the participants who had experience in ophthalmic artificial intelligence. 


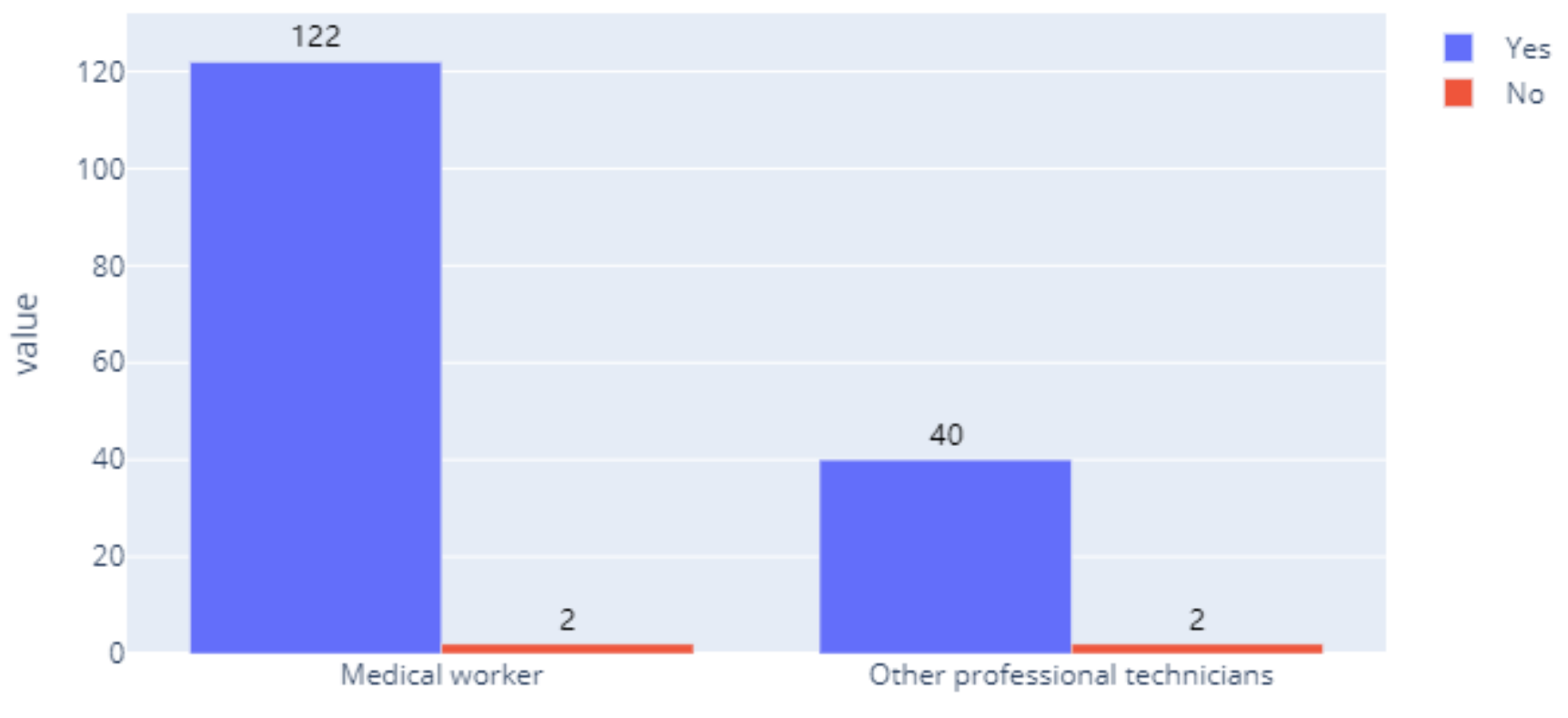

Work area

\section{Figure 9}

Attitudes of the participants whose understanding level was "almost understand" or above toward the question.

\section{Supplementary Files}

This is a list of supplementary files associated with this preprint. Click to download.

- OphthalmologyArtificiallntelligenceQuestionnaireSurvey.docx 